\title{
A geometric approach to accretivity
}

\author{
by \\ LeOnid V. Kovalev (College Station, TX)
}

\begin{abstract}
We establish a connection between generalized accretive operators introduced by F. E. Browder and the theory of quasisymmetric mappings in Banach spaces pioneered by J. Väisälä. The interplay of the two fields allows for geometric proofs of continuity, differentiability, and surjectivity of generalized accretive operators.
\end{abstract}

1. Introduction. Let $X$ and $Y$ be Banach spaces. We only consider Banach spaces over real scalars. Let $\phi: X \rightarrow Y^{*}$ be a norm-preserving 1homogeneous mapping of $X$ into the dual space of $Y$. More precisely, we assume that

$$
\|\phi(x)\|_{Y^{*}}=\|x\|_{X} \quad \text { and } \quad \phi(\lambda x)=\lambda \phi(x)
$$

for all $x \in X$ and $\lambda \geq 0$. We use angle brackets $\langle\cdot, \cdot\rangle$ to denote the pairing of a Banach space with its dual, e.g. $\left\langle x, x^{*}\right\rangle$ stands for $x^{*}(x)$, where $x \in X$ and $x^{*} \in X^{*}$. Other notation: $B_{X}(a, r)=\{x \in X:\|x-a\|<r\}, S(X)=$ $\{x \in X:\|x\|=1\}$, and $\bar{x}=x /\|x\|$, where $x \in X \backslash\{0\}$.

The following definition was introduced by Browder $[4,5]$ to unify the theories of monotone and accretive mappings in Banach spaces.

Definition 1.1. Let $\phi$ be as in (1.1), and let $\Omega$ be a subset of $X$. A mapping $F: \Omega \rightarrow Y$ is called $\phi$-accretive if

$$
\left\langle F\left(x_{1}\right)-F\left(x_{2}\right), \phi\left(x_{1}-x_{2}\right)\right\rangle \geq 0
$$

for all $x_{1}, x_{2} \in \Omega$.

An additional assumption on $\phi$ is common in the literature $[4,5,11,25$, $26,27]$, namely, $\phi(X)$ is assumed to be norm-dense in $Y^{*}$. This assumption will be imposed on $\phi$ when needed. Two special cases of Definition 1.1 are of most interest: $Y=X$ and $Y=X^{*}$. When $Y=X^{*}$ and $\phi$ is the canonical

2000 Mathematics Subject Classification: Primary 47H06; Secondary 47H05, 30C65.

Key words and phrases: accretive, monotone, quasisymmetric mapping, Banach space, metric space.

The author was supported by an NSF Young Investigator award under grant DMS 0601926. 
embedding of $X$ into its second dual $X^{* *}$, a $\phi$-accretive mapping $F: X \rightarrow X^{*}$ is called monotone. When $Y=X$, one can use the Hahn-Banach theorem to find a duality mapping on $X$, namely, $\phi: X \rightarrow X^{*}$ such that (1.1) holds and $\langle x, \phi(x)\rangle=\|x\|^{2}$ for all $x \in X$. This leads to the concept of an accretive mapping $F: X \rightarrow X$. See $[5,7,35]$ for expositions of these topics. The basic problems, motivated by applications to differential equations, are to determine the accretivity-type conditions that imply $F(X)=Y$, or at least that $F(X)$ is dense or closed in $Y$ (e.g. [6]). Such conditions typically involve replacing the right-hand side of (1.2) with a nonnegative term involving $\left\|x_{1}-x_{2}\right\|$ or $\left\|F\left(x_{1}\right)-F\left(x_{2}\right)\right\|$. This paper is concerned with the following modification of Definition 1.1, which goes back to Sobolevskiu [29].

Definition 1.2. Let $X, Y, \Omega$, and $\phi$ be as in Definition 1.1, and let $\delta \in(0,1]$. We say that a mapping $F: \Omega \rightarrow Y$ is $(\phi, \delta)$-accretive if for all $x_{1}, x_{2} \in \Omega$,

$$
\left\langle F\left(x_{1}\right)-F\left(x_{2}\right), \phi\left(x_{1}-x_{2}\right)\right\rangle \geq \delta\left\|F\left(x_{1}\right)-F\left(x_{2}\right)\right\|_{Y}\left\|x_{1}-x_{2}\right\|_{X} .
$$

Definition 1.2 admits a simple geometric interpretation when $X=Y$ is a Hilbert space $\mathcal{H}$ and $\phi$ is the identity on $\mathcal{H}$. Namely, $F: \Omega \rightarrow \mathcal{H}$ is $(\mathrm{Id}, \delta)$-accretive if and only if the vectors $F\left(x_{1}\right)-F\left(x_{2}\right)$ and $x_{1}-x_{2}$ form an angle of at most $\cos ^{-1}(\delta)<\pi / 2$ for all $x_{1}, x_{2} \in \Omega$. This special case was considered in [20]. The general form of (1.3) can also be thought of as an "angle inequality" because it is invariant under rescaling of norms in $X$ and $Y$. Examples of $(\phi, \delta)$-accretive mappings are given in Section 2. Most of our results hold under the following assumptions on $\phi: X \rightarrow Y^{*}$, which include (1.1) for ease of reference:

$$
\begin{aligned}
& \phi(\lambda x)=\lambda \phi(x) \text { for all } \lambda \geq 0 \text { and } x \in X ; \\
& \phi: S(X) \rightarrow S\left(Y^{*}\right) \text { is uniformly continuous. }
\end{aligned}
$$

We write $\omega_{\phi}$ for the modulus of continuity of the restriction of $\phi$ to $S(X)$. If $X$ is uniformly smooth (which is equivalent to $X^{*}$ being uniformly convex by Propositions 1.e.2 and 1.e.3 in [22]), then the duality mapping $\phi: X \rightarrow X^{*}$ satisfies (UC) (see Proposition 12.3 in [7] or Theorem 5.5.10 in [23]). The following is the main result of this paper.

Theorem 1.3. Let $X$ be a Banach space of dimension at least 2. Suppose that $F: B_{X}(0,1) \rightarrow Y$ is a nonconstant $(\phi, \delta)$-accretive mapping with $\phi$ as in (UC). Then for any $r \in(0,1), F$ is $\eta$-quasisymmetric in $B(0, r)$ with $\eta(t)=C \max \left\{t^{\alpha}, t^{1 / \alpha}\right\}$, where $C$ and $\alpha$ depend only on $\delta, r$, and $\omega_{\phi}$.

The definition of quasisymmetric mappings and the proof of Theorem 1.3 are given in Section 3. Some applications of Theorem 1.3 are collected in Section 4 . They concern the continuity, differentiability, and surjectivity of $(\phi, \delta)$-accretive mappings. 
2. Examples. The purpose of this section is to motivate Definition 1.2 by presenting several examples of mappings that satisfy (1.3).

EXAMPLE 2.1. Every linear operator $F: X \rightarrow Y$ is $(\phi, 1)$-accretive, where $\phi(x)$ is defined (via the Hahn-Banach theorem) so that (1.1) holds and $\langle F(x), \phi(x)\rangle=\|F(x)\|\|x\|$ for all $x \in X$.

EXAmPle 2.2. Suppose that $F: X \rightarrow X$ satisfies the Lipschitz condition

$$
\|F(a)-F(b)\| \leq L\|a-b\|, \quad a, b \in X,
$$

for some constant $L$. Then for any $\lambda>L$ the mapping $F_{\lambda}(x):=F(x)+\lambda x$ is $(\phi, \delta)$-accretive, where $\phi$ is a duality mapping for $X$ and $\delta=(\lambda-L) /(\lambda+L)$.

Proof. First observe that

$$
(\lambda-L)\|a-b\| \leq\left\|F_{\lambda}(a)-F_{\lambda}(b)\right\| \leq(\lambda+L)\|a-b\|
$$

for all $a, b \in X$. This and the definition of $\phi$ imply

$$
\begin{aligned}
\left\langle F_{\lambda}(a)-F_{\lambda}(b), \phi(a-b)\right\rangle & =\lambda\|a-b\|^{2}+\langle F(a)-F(b), \phi(a-b)\rangle \\
& \geq(\lambda-L)\|a-b\|^{2} \geq \delta\left\|F_{\lambda}(a)-F_{\lambda}(b)\right\|\|a-b\| .
\end{aligned}
$$

Example 2.2 shows a direct connection between differentiability properties of Lipschitz and $(\phi, \delta)$-accretive mappings, at least when the domain and the target space are the same. See Proposition 4.5 in Section 3 and the remark following it.

EXAMPle 2.3. Let $T$ be a positive invertible operator on a Hilbert space $\mathcal{H}$. Given a number $p>1$, define $A: \mathcal{H} \rightarrow \mathcal{H}$ by

$$
A(x)=\langle T(x), x\rangle^{(p-2) / 2} T(x) .
$$

Then $A$ is (Id, $\delta$ )-accretive, where $\delta>0$ depends on $p$ and $\|T\|\left\|T^{-1}\right\|$.

Proof. The mapping $A$ coincides, up to a constant multiple, with the gradient of the convex function $u(x)=\langle T(x), x\rangle^{p / 2}=\left\|T^{1 / 2}(x)\right\|^{p}$. Convex functions on a Hilbert space with an $(\mathrm{Id}, \delta)$-accretive gradient are called quasiuniformly convex in [20,21]. There it was observed that the function $x \mapsto\|x\|^{p}$ is quasiuniformly convex. Since quasiuniform convexity is preserved under linear isomorphisms [21, p.1040] such as $T^{1 / 2}$, it follows that $A$ is (Id, $\delta)$-accretive.

Example 2.3 arises in the theory of nonlinear elliptic equations and systems [18], in which case $\mathcal{H}$ is the space of alternating $l$-multilinear forms on $\mathbb{R}^{n}$ with $0 \leq l \leq n$. The following example is more general than (2.1), but may seem less intuitive.

ExAmple 2.4. An $\mathcal{A}$-harmonic system of partial differential equations $[9,10]$ involves a nonlinear mapping $\mathcal{A}: \mathbb{R}^{n} \times \mathcal{H} \rightarrow \mathcal{H}$ that satisfies the 
assumptions of local Lipschitz continuity

$$
\|\mathcal{A}(x, \xi)-\mathcal{A}(x, \zeta)\| \leq K\|\xi-\zeta\|(\|\xi\|+\|\zeta\|)^{p-2}
$$

and uniform monotonicity

$$
\langle\mathcal{A}(x, \xi)-\mathcal{A}(x, \zeta), \xi-\zeta\rangle \geq K^{-1}\|\xi-\zeta\|^{2}(\|\xi\|+\|\zeta\|)^{p-2}
$$

with $K, p>1$ independent of the variables $x \in \mathbb{R}^{n}$ and $\xi, \zeta \in \mathcal{H}$. It is easy to see that for any fixed $x$ the mapping $\xi \mapsto \mathcal{A}(x, \xi)$ is (Id, $K^{-2}$ )-accretive.

Further examples and applications can be found in [15, 19, 20, 21, 29].

3. Quasisymmetric behavior of accretive mappings. Quasisymmetric mappings can be defined in a general metric space. An account of their properties in this setting can be found in $[16,30]$. Quasisymmetric embeddings of metric spaces into normed spaces were studied, e.g., in $[1,17,24]$. The studies of quasisymmetric mappings in Banach spaces began with Väisälä's paper [32].

Definition 3.1 ([30]). Let $Y$ and $Z$ be metric spaces. An injective mapping $f: Y \rightarrow Z$ is called quasisymmetric if there is a homeomorphism $\eta:[0, \infty) \rightarrow[0, \infty)$ such that

$$
\frac{d_{Z}(f(y), f(a))}{d_{Z}(f(x), f(b))} \leq \eta\left(\frac{d_{Y}(y, a)}{d_{Y}(y, b)}\right)
$$

for any distinct points $a, b, y \in Y$.

We write that $f$ is $\eta$-quasisymmetric when it is desirable to emphasize the role of $\eta$. Such $f$ must be continuous because of the assumption $\eta(0)=0$. Note that the inverse of $f$ is $\widetilde{\eta}$-quasisymmetric with $\widetilde{\eta}(t)=1 / \eta^{-1}(1 / t)$. Consequently, a quasisymmetric mapping is a homeomorphism onto its image. The main goal of this section is to prove that a nonconstant $(\phi, \delta)$-accretive mapping defined on a convex open subset of $X$ is locally quasisymmetric provided that $\operatorname{dim} X>1$ and $\phi$ is uniformly continuous on the unit sphere of $X$. That the assumption $\operatorname{dim} X>1$ is necessary follows from the fact that any nondecreasing function $f: \mathbb{R} \rightarrow \mathbb{R}$ is (Id, 1 )-accretive. The following example shows that the continuity of $\phi$ is also necessary.

Proposition 3.2. Let $X=\ell_{1}^{n}$ with the standard basis $e_{1}, \ldots, e_{n}$. Given a collection of nondecreasing functions $g_{1}, \ldots, g_{n}: \mathbb{R} \rightarrow \mathbb{R}$ such that $g_{i}(0)=0$, define a mapping $F: X \rightarrow X$ by $F\left(\sum_{i} c_{i} e_{i}\right)=\sum_{i} g_{i}\left(c_{i}\right) e_{i}$. Then $F$ is $(\phi, 1)$ accretive for any duality mapping $\phi: \ell_{1}^{n} \rightarrow \ell_{\infty}^{n}$.

Proof. Let $x=\sum_{i} a_{i} e_{i}$ and $y=\sum_{i} b_{i} e_{i}$. By the definition of $\phi$ we have

$$
\begin{aligned}
& \left\langle e_{i}, \phi(x-y)\right\rangle=\|x-y\| \operatorname{sign}\left(a_{i}-b_{i}\right) \quad \text { if } a_{i} \neq b_{i} ; \\
& \left\langle e_{i}, \phi(x-y)\right\rangle \in[-\|x-y\|,\|x-y\|] \quad \text { if } a_{i}=b_{i} \text {. }
\end{aligned}
$$


Therefore,

$$
\begin{aligned}
\langle F(x)-F(y), \phi(x-y)\rangle & =\sum_{i}\|x-y\| \operatorname{sign}\left(a_{i}-b_{i}\right)\left(g_{i}\left(a_{i}\right)-g_{i}\left(b_{i}\right)\right) \\
& =\|x-y\| \sum_{i}\left|g_{i}\left(a_{i}\right)-g_{i}\left(b_{i}\right)\right| \\
& =\|x-y\|\|F(x)-F(y)\|
\end{aligned}
$$

as required.

The mapping $F$ constructed in Proposition 3.2 need not be quasisymmetric, continuous, onto, or one-to-one. The issue here is that $\ell_{1}^{n}$ does not admit a continuous duality mapping, being a nonsmooth Banach space. The next example illustrates why we need the uniform continuity of $\phi$ to prove that $(\phi, \delta)$-accretive mappings are quasisymmetric.

ExAmple 3.3. Let $\left\{e_{i}: i \geq 1\right\}$ be the standard basis of $\ell_{2}$. Define a linear mapping $F: \ell_{2} \rightarrow \ell_{2}$ by $F\left(e_{i}\right)=e_{i} / i$, and let

$$
\phi(x)=\frac{\|x\|}{\|F(x)\|} F(x), \quad x \in \ell_{2} .
$$

It is easy to see that $\phi$ is continuous on the unit sphere $S\left(\ell_{2}\right)$. The mapping $F$ is $(\phi, 1)$-accretive by the definition of $\phi$, but it is not quasisymmetric since

$$
\frac{\left\|F\left(e_{1}\right)-F(0)\right\|}{\left\|F\left(e_{i}\right)-F(0)\right\|} \rightarrow \infty \quad \text { as } i \rightarrow \infty \text {. }
$$

The proof of Theorem 1.3 requires three lemmas.

LemMA 3.4. Let $\Omega$ be a subset of a Banach space $X$. Let $F: \Omega \rightarrow Y$ be a $(\phi, \delta)$-accretive mapping with $\phi$ as in (UC). Choose $\varepsilon>0$ so that $\omega_{\phi}(\varepsilon) \leq \delta / 2$. If $x, a, b$ are distinct points in $\Omega$ such that

$$
\|\overline{x-a}-\overline{b-a}\| \leq \varepsilon \quad \text { and } \quad\|\overline{x-b}-\overline{a-b}\| \leq \varepsilon,
$$

then

$$
\|F(x)-F(a)\|+\|F(x)-F(b)\| \leq 2 \delta^{-1}\|F(a)-F(b)\| .
$$

Proof. Since $F$ is $(\phi, \delta)$-accretive, we have

$$
\langle F(x)-F(a), \phi(\overline{x-a})\rangle \geq \delta\|F(x)-F(a)\| .
$$

It follows from (3.1) that

$$
|\langle F(x)-F(a), \phi(\overline{x-a})\rangle-\langle F(x)-F(a), \phi(\overline{b-a})\rangle| \leq \frac{\delta}{2}\|F(x)-F(a)\| .
$$

Inequalities (3.3) and (3.4) yield

$$
\langle F(x)-F(a), \phi(\overline{b-a})\rangle \geq \frac{\delta}{2}\|F(x)-F(a)\| .
$$


Replacing (3.3) with $\langle F(b)-F(x), \phi(\overline{b-x})\rangle \geq \delta\|F(b)-F(x)\|$ and arguing as above, we obtain

$$
\langle F(b)-F(x), \phi(\overline{b-a})\rangle \geq \frac{\delta}{2}\|F(b)-F(x)\| .
$$

The sum of (3.5) and (3.6) is

$$
\langle F(b)-F(x), \phi(\overline{b-a})\rangle \geq \frac{\delta}{2}(\|F(x)-F(a)\|+\|F(x)-F(b)\|),
$$

which implies (3.2).

Note that the inequalities (3.1) hold whenever $x$ is a convex combination of $a$ and $b$. The following elementary estimate will be useful in connection with Lemma 3.4 .

Lemma 3.5. If $u, v \in X$ are nonzero vectors such that $\|u-v\|=\tau\|u\|$ with $\tau<1$, then

$$
\|\bar{u}-\bar{v}\| \leq \frac{2 \tau}{1-\tau}
$$

Proof. We may assume $\|u\|=1$. Since $1-\tau \leq\|v\| \leq 1+\tau$, it follows that

$$
\|u-\bar{v}\| \leq \frac{\|u-v\|}{\|v\|}+\frac{|\|v\|-1|}{\|v\|} \leq \frac{2 \tau}{1-\tau}
$$

Definition 3.6. A metric space $Z$ is called c-quasiconvex if there is $c \geq 1$ such that any two points $a, b \in Z$ can be connected by a continuous curve in $Z$ whose length is at most $c d_{Z}(a, b)$.

Definition 3.7. Let $Y$ and $Z$ be metric spaces. A mapping $f: Y \rightarrow Z$ is very weakly quasisymmetric if there is a constant $H$ such that

$$
d_{Z}(f(y), f(a)) \leq H d_{Z}(f(y), f(b)) \quad \text { whenever } \quad d_{Y}(y, a) \leq d_{Y}(y, b) .
$$

If, in addition, $f$ is a homeomorphism of $Y$ onto $f(Y)$, then $f$ is weakly quasisymmetric [30].

Lemma 3.8. Let $\Omega$ be a convex subset of $X$. Let $F: \Omega \rightarrow Y$ be a nonconstant $(\phi, \delta)$-accretive mapping with $\phi$ as in (UC). If $F$ is very weakly quasisymmetric, then it is $\eta$-quasisymmetric with $\eta(t)=C \max \left\{t^{\alpha}, t^{1 / \alpha}\right\}$, where $C$ and $\alpha$ depend only on $\delta$ and the constant $H$ in (3.8).

Proof. Since $F$ is nonconstant, inequality (3.8) implies that $F$ is injective. Our first step is to prove that $F$ is weakly quasisymmetric. Given distinct points $a, b \in \Omega$, let $v=b-a$ and define $h(t)=\langle F(a+t v), \phi(\bar{v})\rangle$ for $0 \leq t \leq 1$. Since $F$ is $(\phi, \delta)$-accretive, it follows that

$$
\delta\|F(a+t v)-F(a+s v)\| \leq h(t)-h(s) \leq\|F(a+t v)-F(a+s v)\|
$$

for $0 \leq s \leq t \leq 1$. Therefore, $h:[0,1] \rightarrow \mathbb{R}$ is very weakly quasisymmetric with the constant $H^{\prime}=\delta^{-1} H$. By Theorem 2.20 of [30] a nonconstant 
very weakly quasisymmetric mapping from an interval into $\mathbb{R}$ is weakly quasisymmetric. Hence $h$ is continuous on $[0,1]$. By (3.9) the restriction of $F$ to the segment $[a, b]$ is continuous as well. By (3.8), $F$ itself must be continuous at every point of $[a, b]$. Since $a$ and $b$ are arbitrary, $F$ is continuous on $\Omega$. Also, its inverse $F^{-1}$ is continuous on $F(\Omega)$ by Theorem 2.20 of [30]. Thus, $F$ is weakly quasisymmetric in $\Omega$.

Next we claim that the set $F(\Omega)$ is $\delta^{-1}$-quasiconvex. Indeed, for any $a, b \in \Omega$ the image of the segment $[a, b]$ under $F$ is a curve connecting $F(a)$ to $F(b)$. The left inequality of (3.9) implies that the length of $F([a, b])$ is at most

$$
\delta^{-1}(h(1)-h(0)) \leq \delta^{-1}\|F(a)-F(b)\|
$$

as claimed.

According to Theorem 6.6 in [33], every weakly quasisymmetric mapping between $c$-quasiconvex spaces is $\widetilde{\eta}$-quasisymmetric with $\widetilde{\eta}$ depending only on $c$ and $H$ in (3.8). Thus $F$ is quasisymmetric. By Theorem 3.10 in [30], every $\widetilde{\eta}$-quasisymmetric mapping with a connected domain is $\eta$-quasisymmetric with $\eta(t)=C \max \left\{t^{\alpha}, t^{1 / \alpha}\right\}$, where $C$ and $\alpha$ depend only on $\widetilde{\eta}$. This completes the proof.

Proof of Theorem 1.3. By virtue of Lemma 3.8 it suffices to prove that $F: B_{X}(0, r) \rightarrow Y$ is very weakly quasisymmetric. First we prove that there exist positive constants $\sigma$ and $H$, depending only on $\delta, r$, and $\omega_{\phi}$, such that

$$
\|F(x)-F(a)\| \leq H\|F(x)-F(b)\|
$$

whenever $x, a, b \in B_{X}(0, r)$ are such that $\|x-a\| \leq \sigma\|x-b\|$.

Choose $\tau>0$ so that $\omega_{\phi}(2 \tau /(1-\tau)) \leq \delta / 2$. Set $M=\left\lfloor 4 \tau^{-1}\right\rfloor+1$ and $\sigma=2^{-M}(1-r)$. Suppose that $a, b, x \in B_{X}(0, r)$ are distinct points such that $\|x-a\| \leq \sigma\|x-b\|$. Let

$$
b^{\prime}=x+2^{M-1}\|a-x\|(\overline{b-x}) .
$$

Since $b^{\prime}$ lies between $x$ and $b$, Lemma 3.4 implies

$$
\left\|F(x)-F\left(b^{\prime}\right)\right\| \leq 2 \delta^{-1}\|F(x)-F(b)\| .
$$

Let $Q$ be a 2-dimensional subspace containing the vectors $a-x$ and $b-x$. Any two points of the unit sphere $S(Q)$ can be connected in $S(Q)$ by a curve of length at most $4[28$, p. 17]. Therefore, there exists a sequence $z_{1}, \ldots, z_{M} \in S(Q)$ such that

$$
z_{1}=\overline{b-x}, \quad z_{M}=\overline{a-x}, \quad\left\|z_{i}-z_{i-1}\right\| \leq \tau \quad \text { for } 2 \leq i \leq M .
$$

We claim that for any $0<c<(1-r) / 2$ and any $i=2, \ldots, M$,

$$
\left\|F(x)-F\left(x+c z_{i}\right)\right\| \leq 2 \delta^{-1}\left\|F(x)-F\left(x+2 c z_{i-1}\right)\right\| .
$$


This follows from Lemma 3.4 applied to the points $x, x+c z_{i}$, and $x+2 c z_{i-1}$, which are all contained in $B_{X}(0,1)$. Indeed, we have

$$
\left\|\overline{c z_{i}}-\overline{2 c z_{i-1}}\right\|=\left\|z_{i}-z_{i-1}\right\| \leq \tau \text {. }
$$

Since $\left\|\left(2 z_{i-1}-z_{i}\right)-z_{i-1}\right\| \leq \tau\left\|z_{i-1}\right\|$, it follows from Lemma 3.5 that

$$
\left\|\overline{2 c z_{i-1}-c z_{i}}-\overline{2 c z_{i-1}}\right\| \leq \frac{2 \tau}{1-\tau} .
$$

By virtue of our choice of $\tau$, Lemma 3.4 implies (3.12).

We shall use (3.12) with $c=c_{i}:=2^{M-i}\|a-x\|$ for $i=2, \ldots, M$. Note that the assumption $c<(1-r) / 2$ holds because

$$
c_{i} \leq c_{2} \leq 2^{M-2} \sigma\|b-x\|=2^{-2}(1-r)\|b-x\|<\frac{1-r}{2} .
$$

Thus we obtain

$$
\begin{aligned}
\|F(x)-F(a)\| & =\left\|F(x)-F\left(x+c_{M} z_{M}\right)\right\| \\
& \leq 2 \delta^{-1}\left\|F(x)-F\left(x+2 c_{M} z_{M-1}\right)\right\| \\
& =2 \delta^{-1}\left\|F(x)-F\left(x+c_{M-1} z_{M-1}\right)\right\| \\
& \leq \cdots \leq 2^{M-1} \delta^{1-M}\left\|F(x)-F\left(x+c_{1} z_{1}\right)\right\| \\
& =2^{M-1} \delta^{1-M}\left\|F(x)-F\left(b^{\prime}\right)\right\| .
\end{aligned}
$$

This and (3.11) yield (3.10).

Finally, suppose that $x, a, b \in B_{X}(0, r)$ are distinct points such that $\|x-a\| \leq\|x-b\|$. If $\|a-b\| \leq \sigma\|x-b\|$, then the triangle inequality and (3.10) yield

$$
\|F(x)-F(a)\| \leq\|F(x)-F(b)\|+\|F(a)-F(b)\| \leq(H+1)\|F(x)-F(b)\| .
$$

Now consider the case $\|a-b\| \geq \sigma\|x-b\|$. If $y$ is a point on the line segment $[x, a]$, then

$$
\begin{aligned}
\|y-b\| & \geq \max \{\|x-b\|-\|x-y\|,\|a-b\|-\|a-y\|\} \\
& \geq \frac{1}{2}(\|x-b\|-\|x-y\|+\|a-b\|-\|a-y\|) \\
& \geq \frac{1}{2}((1+\sigma)\|x-b\|-\|x-a\|) \geq \frac{\sigma}{2}\|x-b\| .
\end{aligned}
$$

Choose a partition $y_{0}, \ldots, y_{N}$ of the segment $[x, a]$ so that $y_{0}=x, y_{N}=a$, and $\left\|y_{j}-y_{j-1}\right\| \leq \sigma^{2}\|x-b\| / 2$ for $1 \leq j \leq N$. Note that $N$ depends only on $\sigma$. For $j=1, \ldots, N$ we have

$$
\begin{aligned}
\left\|F\left(y_{j}\right)-F(b)\right\| & \leq\left\|F\left(y_{j}\right)-F\left(y_{j-1}\right)\right\|+\left\|F\left(y_{j-1}\right)-F(b)\right\| \\
& \leq(H+1)\left\|F\left(y_{j-1}\right)-F(b)\right\|,
\end{aligned}
$$


where the last inequality follows from (3.10). Therefore,

$$
\|F(a)-F(b)\| \leq(H+1)^{N}\|F(x)-F(b)\|,
$$

whence $F$ is very weakly quasisymmetric with the constant $(H+1)^{N}+1$.

If $F$ is defined on the whole space $X$, an obvious rescaling argument shows that $F$ is globally quasisymmetric. We state this as a corollary.

Corollary 3.9. Let $X$ be a Banach space of dimension at least 2. Suppose that $F: X \rightarrow Y$ is a nonconstant $(\phi, \delta)$-accretive mapping with $\phi$ as in (UC). Then $F$ is $\eta$-quasisymmetric in $X$ with $\eta(t)=C \max \left\{t^{\alpha}, t^{1 / \alpha}\right\}$, where $C$ and $\alpha$ depend only on $\delta$ and $\omega_{\phi}$.

In contrast to Corollary 3.9, for every $\delta \in(0,1)$ there exist a bounded open convex set $\Omega \subset \mathbb{R}^{2}$ and an (Id, $\delta$ )-accretive mapping $F: \Omega \rightarrow \mathbb{R}^{2}$ that is not quasisymmetric in $\Omega$. See Example 15 in [20].

4. Applications. Most of the results in this section are based on Theorem 1.3.

Proposition 4.1. Let $X$ be a Banach space of dimension at least 2. If (UC) holds, then every nonconstant $(\phi, \delta)$-accretive mapping $F: X \rightarrow X$ is locally Hölder continuous with an exponent $\alpha \in(0,1)$. Furthermore, there exists $C>0$ such that

$$
C^{-1} \min \left\{\|x\|^{\alpha},\|x\|^{1 / \alpha}\right\} \leq\|F(x)-F(0)\| \leq C \max \left\{\|x\|^{\alpha},\|x\|^{1 / \alpha}\right\}
$$

for all $x \in X$.

Proof. Choose a point $b \in X$ so that $\|b\|=1$, and apply Corollary 3.9. The constant $C$ will depend on $\|F(0)-F(b)\|$ in addition to $\phi$ and $\delta$.

Under the assumptions of Theorem 1.3 the range of $F$ is closed in $Y$, because the image of a complete metric space under a quasisymmetric mapping is itself complete [30, 2.24]. However, $F$ need not be surjective if $Y$ is infinite-dimensional. Indeed, let $F: \ell_{2} \rightarrow \ell_{2}$ be the forward shift operator. It is clear that $F$ is $(F, 1)$-accretive, hence quasisymmetric, but it is not surjective. Proposition 4.2 shows that $(\phi, \delta)$-accretive mappings are surjective provided that $\phi$ has dense image and $Y$ admits an equivalent uniformly smooth norm. A norm on $Y$ is called uniformly smooth if its modulus of smoothness, defined by

$$
\varrho_{Y}(\tau)=\frac{1}{2} \sup \left\{\left\|y+y^{\prime}\right\|+\left\|y-y^{\prime}\right\|-2:\|y\| \leq 1,\left\|y^{\prime}\right\| \leq \tau\right\},
$$

satisfies $\varrho_{Y}(\tau) / \tau \rightarrow 0$ as $\tau \rightarrow 0$. Banach spaces that admit an equivalent uniformly smooth norm are known as superreflexive [2, Thm. A.6].

Proposition 4.2. Let $\Omega$ be an open subset of a Banach space $X$, where $\operatorname{dim} X \geq 2$. Let $Y$ be a superreflexive Banach space. Suppose, in addition 
to (UC), that

$$
\phi(X) \text { is norm-dense in } Y^{*} .
$$

Then every nonconstant $(\phi, \delta)$-accretive mapping $F: \Omega \rightarrow Y$ is open. Moreover, if $\Omega=X$, then $F$ is surjective.

Lemma 4.3. Suppose that $F: \Omega \rightarrow Y$ is a $(\phi, \delta)$-accretive mapping, where $\phi$ satisfies (UC) and (D). Let $\|\cdot|\||$ be a norm on $Y$ that is equivalent to the original norm $\|\cdot\|$. Then $F: \Omega \rightarrow(Y,\|\cdot \cdot\|)$ is $(\widetilde{\phi}, \widetilde{\delta})$-accretive, where $\widetilde{\phi}$ satisfies (UC) and (D) with respect to $\|\cdot \cdot\|$.

Proof. The norm $\||\cdot|\|_{Y}$ induces a norm $\|\cdot \mid\|_{*}$ on the dual space of $Y$. Let $\widetilde{\phi}=\psi \circ \phi$, where $\psi: Y^{*} \rightarrow Y^{*}$ is defined by $\psi(y)=\left(\|y\| /\|y\|_{*}\right) y$ for $y \neq 0$, and $\psi(0)=0$. Since $\psi$ is bi-Lipschitz, it follows that $\widetilde{\phi}$ satisfies (UC) and (D). Also,

$$
\begin{aligned}
\left\langle F\left(x_{1}\right)-F\left(x_{2}\right), \widetilde{\phi}\left(x_{1}-x_{2}\right)\right\rangle & =\frac{\left\|\phi\left(x_{1}-x_{2}\right)\right\|}{\left\|\phi\left(x_{1}-x_{2}\right)\right\|_{*}}\left\langle F\left(x_{1}\right)-F\left(x_{2}\right), \phi\left(x_{1}-x_{2}\right)\right\rangle \\
& \geq \frac{\left\|\phi\left(x_{1}-x_{2}\right)\right\|}{\left\|\phi\left(x_{1}-x_{2}\right)\right\|_{*}} \delta\left\|F\left(x_{1}\right)-F\left(x_{2}\right)\right\|\left\|x_{1}-x_{2}\right\| \\
& \geq \widetilde{\delta}\left\|F\left(x_{1}\right)-F\left(x_{2}\right)\right\|\left\|x_{1}-x_{2}\right\|
\end{aligned}
$$

with $\widetilde{\delta}>0$ independent of $x_{1}, x_{2} \in X$.

Proof of Proposition 4.2. The first part of the statement implies the second part because $F(X)$ is closed in $Y$. By virtue of Lemma 4.3 we may assume that $Y$ is given a uniformly smooth norm $\|\cdot\|$ with the modulus of smoothness $\varrho_{Y}$. Since $\varrho_{Y}(\tau) / \tau \rightarrow 0$ as $\tau \rightarrow 0$, there exists $\tau_{0} \in(0,1)$ such that $\varrho_{Y}\left(\tau_{0}\right) \leq \delta \tau_{0} / 4$.

It suffices to show that $F(\Omega)$ is open. Given $x_{0} \in \Omega$, choose an open ball $B=B\left(x_{0}, r\right)$ so that $B\left(x_{0}, 2 r\right) \subset \Omega$. By Theorem 1.3 the restriction of $F$ to $\bar{B}$ is quasisymmetric, hence $F(\partial B)$ is closed. Let $R$ be the distance from $y_{0}:=F\left(x_{0}\right)$ to the set $F(\partial B)$. We shall prove that $B\left(y_{0}, R / 4\right) \subset F(\bar{B})$.

Suppose to the contrary that there is a point $y \in B\left(y_{0}, R / 4\right) \backslash F(\bar{B})$. Replacing $F$ with $F-y$, we may assume that $y=0$. The distance $d:=$ $\operatorname{dist}(0, F(\bar{B}))$ satisfies $0<d \leq\left\|y_{0}\right\|<R / 4$. Choose a point $z \in F(\bar{B})$ such that

$$
\|z\|<d\left(1-\delta \tau_{0} / 4\right)^{-1} \text { and }\|z\|<R / 4 .
$$

By virtue of (D) there exists $v \in S(X)$ such that

$$
\langle-z, \phi(v)\rangle>\left(1-\delta \tau_{0} / 4\right)\|z\| \text {. }
$$

For $t \geq 0$ let $x(t)=F^{-1}(z)+t v$. For some $t^{\prime}>0$ we have $x\left(t^{\prime}\right) \in \partial B$, hence $\left\|F\left(x\left(t^{\prime}\right)\right)-z\right\|>R / 2>\|z\|$. It follows that there is $t_{0} \in\left(0, t^{\prime}\right)$ such 
that $\left\|F\left(x\left(t_{0}\right)\right)-z\right\|=\tau_{0}\|z\|$. Let $z^{\prime}=F\left(x\left(t_{0}\right)\right)-z$. Using (4.2) and the $(\phi, \delta)$-monotonicity of $F$, we obtain

$$
\left\langle z^{\prime}-z, \phi(v)\right\rangle>\delta\left\|z^{\prime}\right\|+\left(1-\delta \tau_{0} / 4\right)\|z\|,
$$

hence

$$
\left\|z-z^{\prime}\right\|>\left(1+3 \delta \tau_{0} / 4\right)\|z\| .
$$

By the uniform smoothness of $Y$,

$$
\left\|z+z^{\prime}\right\|+\left\|z-z^{\prime}\right\| \leq 2\|z\|\left(1+\varrho_{Y}\left(\tau_{0}\right)\right) \leq\|z\|\left(2+\delta \tau_{0} / 2\right) .
$$

Combine (4.3), (4.4), and (4.1) to obtain

$$
\left\|F\left(x\left(t_{0}\right)\right)\right\|=\left\|z+z^{\prime}\right\|<\|z\|\left(1-\delta \tau_{0} / 4\right)<d,
$$

which contradicts the definition of $d$.

Question 4.4. Is the superreflexivity of $Y$ necessary in Proposition 4.2?

A quasisymmetric mapping of a finite-dimensional space into itself is Fréchet differentiable outside of a set of Lebesgue measure zero. This property is no longer true in infinite-dimensional spaces. Indeed, by Corollary 4.8 in [34] for any $\alpha \in(0,1)$ there exists a mapping $F: \ell_{2} \rightarrow \ell_{2}$ such that $\|F(a)-F(b)\|=\|a-b\|^{\alpha}$ for all $a, b \in \ell_{2}$. Although $F$ is quasisymmetric with $\eta(t)=t^{\alpha}$, it does not have any directional derivatives at any point. Moreover, the local Lipschitz constant

$$
L_{F}(a):=\limsup _{x \rightarrow a} \frac{\|F(x)-F(a)\|}{\|x-a\|}
$$

is infinite for all $a \in X$. This can be contrasted with the following

Proposition 4.5. Suppose that $F: X \rightarrow Y$ is $(\phi, \delta)$-accretive and (UC) holds. Then

(i) $L_{F}(x)<\infty$ for all $x \in X \backslash E$, where $E$ is a Borel set which meets every line in a set of linear measure zero.

(ii) If , in addition, $X$ is separable and $Y$ has the Radon-Nikodym property [8], then $F$ is Gateaux differentiable on $X \backslash A$, where $A$ is an Aronszajn null set [2].

Proof. Both results are standard in the case $\operatorname{dim} X=1$ [14, Thm. 3.23]. Let us assume $\operatorname{dim} X>1$.

(i) It is straightforward to verify that the set $E:=\left\{x \in X: L_{F}(x)=\infty\right\}$ is of type $G_{\delta}$. Consider a line $l$ with the parametric equation $t \mapsto a+t v$, where $a \in X, v \in S(X)$. Since the function

$$
g(t):=\langle F(a+t v), \phi(v)\rangle
$$

is increasing, it is differentiable outside of a set of measure zero. By Theorem $1.3, F$ is $\eta$-quasisymmetric for some $\eta$. If $t_{0}$ is a point of differentiability 
of $g$, then

$$
\begin{aligned}
L_{F}\left(a+t_{0} v\right) & \leq \eta(1) \limsup _{t \rightarrow t_{0}} \frac{\left\|F(a+t v)-F\left(a+t_{0} v\right)\right\|}{\left\|t-t_{0}\right\|} \\
& \leq \delta^{-1} \eta(1) \limsup _{t \rightarrow t_{0}} \frac{g(t)-g\left(t_{0}\right)}{t-t_{0}}<\infty .
\end{aligned}
$$

Thus $E \cap l$ has linear measure zero.

(ii) follows from (i) and the Banach space version of the RademacherStepanoff theorem proved by Bongiorno ([3], see also [13]). One can also deduce (ii) from a theorem of Duda [12]. Indeed, fix a unit vector $z \in X$ and choose $\varepsilon$ so that $\omega_{\phi}(\varepsilon) \leq \delta / 2$. Consider two closed convex cones

$$
\begin{aligned}
& K_{1}=\{0\} \cup\{x \in X \backslash\{0\}:\|\bar{x}-z\| \leq \varepsilon\}, \\
& K_{2}=\left\{y \in Y:\langle y, \phi(z)\rangle \geq \frac{\delta}{2}\|y\|\right\} .
\end{aligned}
$$

It is easy to see that $F$ is $\left(K_{1}, K_{2}\right)$-monotone in the sense that $x_{1}-x_{2}$ $\in K_{1}$ implies $F\left(x_{1}\right)-F\left(x_{2}\right) \in K_{2}$. By Corollary 5.4 in [12], $F$ is Gateaux differentiable outside of an Aronszajn null set.

For any fixed $\phi$ and $\delta$ the class of $(\phi, \delta)$-accretive mappings is closed under pointwise convergence. Therefore, the Gateaux derivative of $F$ at $x \in X$, denoted $D_{F}^{x}$, is a $(\phi, \delta)$-accretive mapping whenever $D_{F}^{x}$ exists. If $\phi$ satisfies (UC), then $D_{F}^{x}$ is either zero or a quasisymmetric linear mapping, which is simply an isomorphism into $Y$. Under the assumptions of Proposition 4.2 the derivative $D_{F}^{x}$ is either zero or an isomorphism onto $Y$. This raises the question: under the assumptions of Proposition 4.5(ii), does $F$ have a nonzero Gateaux derivative at some point? The existence of a surjective Gateaux derivative is also unknown when $(\phi, \delta)$-accretivity is replaced with the bi-Lipschitz condition [2, Problem 7.1]. Although in the latter case $D_{F}^{x}$ is always an isomorphism into $Y$, its surjectivity remains an issue.

REMARK 4.6. An injective mapping $F: X \rightarrow X$ is said to be of class QSA if $F$ is quasisymmetric and there is $c>0$ such that

$$
\left\langle F\left(x_{1}\right)-F\left(x_{2}\right), \phi\left(x_{1}-x_{2}\right)\right\rangle \geq c\left\|F\left(x_{1}\right)-F\left(x_{2}\right)\right\|\left\|x_{1}-x_{2}\right\|
$$

for any $x_{1}, x_{2} \in X$ and for any duality mapping $\phi: X \rightarrow X^{*}$. This class of mappings was used in [19] to settle an open question concerning conformal dimension of metric spaces [31]. Theorem 1.3 shows that in uniformly smooth spaces the condition that $F$ is quasisymmetric can be removed, because it follows from (4.5).

Acknowledgments. Thanks are due to the referee for many valuable comments and suggestions. 


\section{References}

[1] P. Assouad, Plongements lipschitziens dans $\mathbb{R}^{n}$, Bull. Soc. Math. France 111 (1983), 429-448.

[2] Y. Benyamini and J. Lindenstrauss, Geometric Nonlinear Functional Analysis, Amer. Math. Soc., Providence, RI, 2000.

[3] D. Bongiorno, Stepanoff's theorem in separable Banach spaces, Comment. Math. Univ. Carolin. 39 (1998), 323-335.

[4] F. E. Browder, Normal solvability and $\phi$-accretive mappings of Banach spaces, Bull. Amer. Math. Soc. 78 (1972), 186-192.

[5] - Nonlinear Operators and Nonlinear Equations of Evolution in Banach Spaces, Proc. Sympos. Pure Math. 18, Part 2, Amer. Math. Soc., Providence, RI, 1976.

[6] A. Buica and A. Domokos, Nearness, accretivity, and the solvability of nonlinear equations, Numer. Funct. Anal. Optim. 23 (2002), 477-493.

[7] K. Deimling, Nonlinear Functional Analysis, Springer, Berlin, 1985.

[8] J. Diestel and J. J. Uhl, Vector Measures, Math. Surveys 15, Amer. Math. Soc., Providence, RI, 1977.

[9] L. D'Onofrio and T. Iwaniec, The p-harmonic transform beyond its natural domain of definition, Indiana Univ. Math. J. 53 (2004), 683-718.

[10] - - , Notes on p-harmonic analysis, in: The $p$-Harmonic Equation and Recent Advances in Analysis, P. Poggi-Corradini (ed.), Contemp. Math. 370, Amer. Math. Soc., Providence, RI, 2005, 25-49.

[11] D. J. Downing and W. O. Ray, Renorming and the theory of $\phi$-accretive set-valued mappings, Pacific J. Math. 106 (1983), 73-85.

[12] J. Duda, Cone monotone mappings: continuity and differentiability, Nonlinear Anal., to appear.

[13] —, On Gateaux differentiability of pointwise Lipschitz mappings, Canad. Math. Bull., to appear.

[14] G. B. Folland, Real Analysis, 2nd ed., Wiley, New York, 1999.

[15] K. E. Gustafson and D. K. M. Rao, Numerical Range. The Field of Values of Linear Operators and Matrices, Springer, New York, 1997.

[16] J. Heinonen, Lectures on Analysis on Metric Spaces, Springer, New York, 2001.

[17] —, Geometric embeddings of metric spaces, Rep. Univ. Jyväskylä Dept. Math. Stat. 90, Jyväskylä, 2003.

[18] T. Iwaniec, p-Harmonic tensors and quasiregular mappings, Ann. of Math. (2) 136 (1992), 589-624.

[19] L. V. Kovalev, Conformal dimension does not assume values between zero and one, Duke Math. J. 134 (2006), 1-13.

[20] -, Quasiconformal geometry of monotone mappings, J. London Math. Soc., to appear.

[21] L. V. Kovalev and D. Maldonado, Mappings with convex potentials and the quasiconformal Jacobian problem, Illinois J. Math. 49 (2005), 1039-1060.

[22] J. Lindenstrauss and L. Tzafriri, Classical Banach Spaces. II. Springer, Berlin, 1996.

[23] R. E. Megginson, An Introduction to Banach Space Theory, Springer, New York, 1998.

[24] A. Naor, Y. Rabani, and A. Sinclair, Quasisymmetric embeddings, the observable diameter, and expansion properties of graphs, J. Funct. Anal. 227 (2005), 273-303.

[25] J. A. Park and S. Park, Surjectivity of $\phi$-accretive operators, Proc. Amer. Math. Soc. 90 (1984), 289-292. 
[26] S. Park and J. A. Park, Surjectivity of generalized $\phi$-accretive operators, in: Nonlinear Functional Analysis and its Applications, F. E. Browder (ed.), Proc. Sympos. Pure Math. 45, Part 2, Amer. Math. Soc., Providence, RI, 1986, 255-259.

[27] W. O. Ray, Phi-accretive operators and Ekeland's theorem, J. Math. Anal. Appl. 88 (1982), 566-571.

[28] J. J. Schäffer, Geometry of Spheres in Normed Spaces, Lecture Notes in Pure Appl. Math. 20, Dekker, New York, 1976.

[29] P. E. Sobolevski1,, On equations with operators forming an acute angle, Dokl. Akad. Nauk SSSR (N.S.) 116 (1957), 754-757 (in Russian).

[30] P. Tukia and J. Väisälä, Quasisymmetric embeddings of metric spaces, Ann. Acad. Sci. Fenn. Ser. A I Math. 5 (1980), 97-114.

[31] J. T. Tyson, Sets of minimal Hausdorff dimension for quasiconformal maps, Proc. Amer. Math. Soc. 128 (2000), 3361-3367.

[32] J. Väisälä, Free quasiconformality in Banach spaces. I, Ann. Acad. Sci. Fenn. Ser. A I Math. 15 (1990), 355-379.

[33] - , The free quasiworld. Freely quasiconformal and related maps in Banach spaces, in: Quasiconformal Geometry and Dynamics, B. Bojarski et al. (eds.), Banach Center Publ. 48, Inst. Math., Polish Acad. Sci., Warszawa, 1999, 55-118.

[34] J. H. Wells and L. R. Williams, Embeddings and Extensions in Analysis, Springer, Berlin, 1975.

[35] E. Zeidler, Nonlinear Functional Analysis and its Applications, Springer, New York, 1990.

Department of Mathematics, Mailstop 3368

Texas A\&M University

College Station, TX 77843-3368, U.S.A.

E-mail: lkovalev@math.tamu.edu

Received September 25, 2006

Revised version April 23, 2007 